\title{
On-Line Estimation of the Maximum Specific Growth Rate of Nitrifiers in Activated Sludge Systems
}

\author{
Zhiguo Yuan, ${ }^{1,3}$ Herwig Bogaert, ${ }^{2,4}$ Martijn Devisscher, ${ }^{3,4}$ \\ Peter Vanrolleghem, ${ }^{3}$ Willy Verstraete ${ }^{4}$ \\ ${ }^{1}$ The Advanced Wastewater Management Centre, The University of \\ Queensland, QLD 4072, Australia; telephone: 0061(7)3365 4374; fax: \\ 0061(7)3365 4726; e-mail: zhiguo@cheque.uq.edu.au \\ ${ }^{2} R \& D$, Aquafin nv., Dijkstraat 8, B2630 Aartselaar, Belgium \\ ${ }^{3}$ Biomath Department, University Gent, Coupure 653, 9000 Gent, Belgium \\ ${ }^{4}$ Department of Microbial Ecology, University Gent, Coupure 653, 9000 \\ Gent, Belgium
}

Received 18 November 1998; accepted 4 May 1999

\begin{abstract}
The on-line estimation of the maximum specific growth rate of autotrophic biomass is addressed in this article. A general nitrification process model, which is valid for any realistic flow pattern, is used to develop the estimation algorithm. Depending on the measurements available, two estimation equations are derived. While both require measuring the nitrification activity of the activated sludge, one requires the additional measurement of the nitrifiable nitrogen concentrations at the two ends of the bioreactor, and the other requires the nitrate nitrogen concentrations at the same locations. The algorithm also requires some stoichiometric and kinetic parameters. However, sensitivity analysis shows that the estimate is insensitive to the parameters other than the autotrophic decay rate. Compared to the existing algorithms, the algorithm developed in this article does not rely on the assumption of ideal flow pattern in the plant and does not require an error-prone estimate of the autotrophic biomass concentration. Experimental and simulation studies show that the algorithm performs well and is robust to influent variations and accidental sludge losses. (C) 1999 John Wiley \& Sons, Inc. Biotechnol Bioeng 65: 265-273, 1999.
\end{abstract}

Keywords: activated sludge; nitrification; nitrifiers; growth rate; estimation; on-line

\section{INTRODUCTION}

The maximum specific growth rate of the autotrophic biomass $\left(\mu_{A, \max }\right)$ plays an important role in the design and operation of a nitrifying activated sludge wastewater treatment plant. The direct determination of this parameter typically involves experiments lasting for a few weeks. Beccari et al. (1979) determined $\mu_{A, \max }$ with the so-called discontinuous technique, in which $\mu_{A, \max }$ is calculated based on the experimentally determined minimum sludge age that allows nitrification. Hall (1974) estimated $\mu_{A, \max }$ by examining the nitrification development process of a barely ni-

Correspondence to: Zhiguo Yuan

Contract grant sponsors: Aartselaar, Belgium; Severn Trent Water, Birmingham, United Kingdom; Fund for Scientific Research.

Contract grant number: G.0286.96 trifying lab-scale fill-and-draw plant after increasing the sludge age to a level at which nitrification becomes possible. $\mu_{A, \max }$ was obtained by plotting the natural logarithm of the nitrate concentration versus time. Similar work was also reported by Antoniou et al. (1990) and Sözen et al. (1996). In their work, the experiments were conducted in a batch reactor, instead of a fill-and-draw plant, where an initial small amount of nitrifiers was seeded. $\mu_{A \text {, max }}$ was again obtained by making use of the time trajectory of the oxidized nitrogen concentration. Some researchers also investigated determining $\mu_{A, \max }$ by using a pure culture of nitrifiers (e.g., Srinath et al., 1976; Hall and Murphy, 1980), with the assumption that the activity of the pure culture is representative of the mixed culture.

The $\mu_{A, \max }$ determined with the above approaches is central to the design of the sludge age of a nitrifying activated sludge wastewater treatment plant (EPA, 1993). It is of limited use, however, for the operation and control of the plant due to the long delay imposed by the experiments. Researches have been devoted in recent years to the on-line estimation of this parameter. Instead of estimating the parameter based on the data obtained from controlled laboratory experiments, on-line estimation algorithms derive the parameter value from the on-line measured process variables of the treatment plant using process models and advanced mathematical techniques. Larrea et al. (1992) used an Extended Kalman Filter algorithm to estimate $\mu_{A, \max }$, together with some other kinetic and stoichiometric parameters, using the IAWQ activated sludge model no. 1 (Henze et al., 1987) and a number of COD, nitrogen, and OUR measurements. Based on the same model and similar measurement conditions, Kabouris et al. (1996) estimated $\mu_{A \text {, max }}$, among other model parameters, using a linearized maximum likelihood algorithm. Marsili-Libelli and Giovannini (1997) used a two-step approach to estimate the specific growth rate of nitrifiers $\left(\mu_{A}\right)$ which is proportional to $\mu_{A \text {, max }}$. In the first step, the nitrifier concentration is estimated using an asymptotically stable observer based on the measurement of nitrate nitrogen concentration. In the sec- 
ond step, $\mu_{A}$ is estimated using a classical recursive leastsquares algorithm. These approaches assume ideal flow patterns in the plant. Reactors are assumed to be completely mixed, and settlers are assumed to be ideal water/sludge separators (point settler). These assumptions are often difficult to justify in reality.

While not directly measurable, $\mu_{A, \max }$ appears in some measurable variables. For example, the maximum autotrophic oxygen uptake rate, which is $\mu_{A, \max } \mathrm{X}_{\mathrm{A}}\left(4.57-Y_{A}\right) / Y_{A}$ (for symbols, see Nomenclature), can be measured by a respirometer (see, e.g., Spanjers and Vanrolleghem, 1995). The maximum nitrogen oxidation rate, which is $\mu_{A, \max } X_{A} /$ $Y_{A}$, can be measured by a titrimetric sensor (Gernaey et al., 1998). Obviously, $\mu_{A, \max }$ can be calculated from these measurements if $X_{A}$ and $Y_{A}$ are known. Nowak and Svardal (1993) and Nowak et al. (1994) used this approach to estimate $\mu_{A \text {,max }}$. The nitrifier concentration was estimated with an asymptotically stable predictor fed with information about the nitrate nitrogen concentration in the plant. Due to the slow convergence of the predictor, this scheme suffers the problem of a slow start-up. The estimate of $X_{A}$ becomes reliable only after the initial transient process of the predictor, which takes about 2 weeks or more (Nowak et al., 1994; Marsilli-Libelli and Giovannini, 1997). This becomes a more severe problem when a plant suffers accidental nitrifier losses due to, for example, spillages of toxic substances or solids wash-out from the settler, since each loss results in an unknown initial nitrifier concentration (Beck, 1981). Another problem is that it is difficult to determine the amount of nitrifiers that are kept in the settler due to the uneven distribution of sludge below the sludge blanket. Neglecting this amount, as done in Nowak et al. (1994), will result in a biased estimate.

Based on a general mathematical model, which is capable of characterizing the nitrification process regardless of the flow pattern in the plant, a new $\mu_{A, \max }$ estimation algorithm is derived in this article. Depending on the signals available, the algorithm has two variants. One uses the nitrification activity of the sludge and the nitrifiable nitrogen concentration at the two ends of the bioreactor, the other uses the same activity signal together with the nitrate nitrogen concentration at the same two locations. Sensitivities of the algorithm to the required stoichiometric and kinetic parameters are analyzed. The impact of the measurement noise on the estimation is also discussed. The algorithm is evaluated by both experimental and simulation studies, and the results are compared to those produced by the algorithm developed in Nowak and Svardal (1993) and Nowak et al. (1994).

\section{A UNIFIED PROCESS MODEL FOR A NITRIFYING REACTOR}

To derive the model, first consider a plug flow aerobic reactor as shown in Figure 1 (for symbols, see Nomenclature). To simplify the derivation, anoxic zones, which may also exist in the plant, are not considered here. However, with minor modifications, the algorithms developed below are also applicable to plants with anoxic zones.

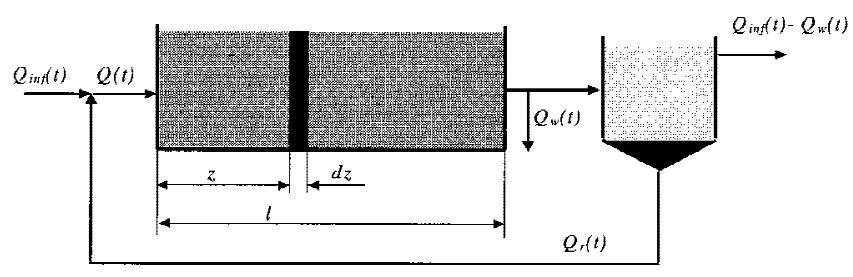

Figure 1. A WWTP with a plug flow reactor.

By making mass balances for the autotrophic biomass, the nitrifiable nitrogen, and the nitrate nitrogen, one easily obtains the following model (for symbols, see Nomenclature):

$$
\begin{gathered}
\frac{\partial X_{A}(z, t)}{\partial t}=-\frac{Q(t)}{A} \frac{\partial X_{A}(z, t)}{\partial z}+\mu_{A}(z, t) X_{A}(z, t)-b_{A} X_{A}(z, t) \\
\frac{\partial S_{N N}(z, t)}{\partial t}=-\frac{Q(t)}{A} \frac{\partial S_{N N}(z, t)}{\partial z}-\frac{\mu_{A}(z, t)}{Y_{A}} X_{A}(z, t)-r_{X B}(z, t) \\
\frac{\partial S_{N O}(z, t)}{\partial t}=-\frac{Q(t)}{A} \frac{\partial S_{N O}(z, t)}{\partial z}+\frac{\mu_{A}(z, t)}{Y_{A}} X_{A}(z, t)-r_{D}(z, t) \\
\mu_{A}(z, t)=\mu_{A, \max } v\left(S_{N H}(z, t), S_{O}(z, t)\right)
\end{gathered}
$$

where the last equation denotes that $\mu_{A}(z, t)$ is linearly dependent on $\mu_{A \text {, max }}$ with a coefficient that is a function (denoted as $v$ ) of $S_{N H}(z, t)$ and $S_{O}(z, t)$. By assuming a point settler (cases where this is not valid will be discussed later) and that there is no autotrophic biomass in the influent, the following boundary conditions can be obtained:

$$
\begin{aligned}
X_{A}(0, t) & =\left(1-\frac{Q_{w}(t)}{Q(t)}\right) X_{A}(l, t) \\
S_{N N}(0, t) & =\frac{Q_{\text {inf }}(t) S_{N N, \text { inf }}(t)+Q_{r}(t) S_{N N}(l, t)}{Q(t)} \\
S_{N O}(0, t) & =\frac{Q_{\text {inf }}(t) S_{N O, \text { inf }}(t)+Q_{r}(t) S_{N O}(l, t)}{Q(t)}
\end{aligned}
$$

Obviously, the influent to the treatment plant is introduced to model (1) via the above boundary conditions. Due to the short hydraulic retention time in the reactor, which is typically a few hours (remembering that $Q$ is the sum of $Q_{\text {inf }}, Q_{r}$ and, for a predenitrification plant, $\left.Q_{\text {int }}\right), X_{A}(z, t)$ does not vary much along the length of the reactor. In fact, according to equation (2), the ratio between $X_{A}(0, t)$ and $X_{A}(l, t)$ is $1-Q_{w}(t) / Q(t)$, which is approximately one since $Q_{w}(t)$ is much smaller than $Q(t)$. As such, it can be assumed that the autotrophic biomass is uniformly distributed in the reactor, i.e., $X_{A}(z, t) \cong X_{A}(t), \forall z \in[0, l]$. Integrating both sides of the first equation in model (1) from 0 to $l$ with respect to $z$ and introducing the boundary constraint into it, one obtains:

$$
\frac{d X_{A}(t)}{d t}=\left[\frac{1}{l} \int_{0}^{l} \mu(z, t) d z\right] \cdot X_{A}(t)-b_{A} X_{A}(t)-\frac{Q_{w}(t)}{V} X_{A}(t)
$$


where $V=A \cdot l$ is the volume of the reactor. Equation (3) is apparently simpler and easier to handle than its origin. When model (1) is referred to in the sequel, it is always meant that the first equation has been replaced by equation (3).

Although model (1) is derived from a plug flow reactor, it is also capable of characterizing reactors with other flow patterns. For a completely mixed reactor, one may simply consider it as a special plug flow reactor with an infinitely small length $l$ and an infinitely large sectional area $A$, satisfying, however, $A \cdot l=V$. As such, the independent variable $z$ in model (1) refers only to one point, say $z_{0}$. By making use of the boundary constraint (2), one easily gets,

$$
\begin{aligned}
& \left.\frac{Q(t)}{A} \frac{\partial S_{N N}(z, t)}{\partial z}\right|_{z=z_{0}}=\frac{Q_{\text {inf }}(t)}{V}\left(S_{N N}\left(z_{0}, t\right)-S_{N N, \text { inf }}(t)\right) \\
& \left.\frac{Q(t)}{A} \frac{\partial S_{N O}(z, t)}{\partial z}\right|_{z=z_{0}}=\frac{Q_{\text {inf }}(t)}{V}\left(S_{N O}\left(z_{0}, t\right)-S_{N O, \text { inf }}(t)\right)
\end{aligned}
$$

In deriving the above equations, it is assumed that the nitrifiable and nitrate nitrogen concentrations in the recycled activated sludge (RAS) are equal to those in the bioreactor. Replacing the transport term in model (1) with (4), it reduces to the standard model for a completely mixed reactor by eliminating the superfluous $z_{0}$. Similarly, a reactor with a series of completely mixed zones can also be described by model (1). In this case, variable $z$ is a set of discrete positions. The fact that model (1) is general for different flow patterns makes the derivations in the sequel valid for all practical reactors.

\section{THE ALGORITHM FOR $\mu_{A, \max }$ ESTIMATION}

Define,

$$
r_{N}(t)=\mu_{A, \max } X_{A}(t) / Y_{A}
$$

$r_{N}(t)$ is called the nitrification activity of the sludge here. The following equation can be obtained by integrating both sides of equation (3) w.r.t. $t$ for a time interval $[t-D, t]$, assuming that $\mu_{A, \max }$ remains constant in $[t-D, t]$ :

$$
\begin{gathered}
\frac{\mu_{A, \max }}{l} \int_{t-D}^{t}\left[\int_{0}^{l} v\left(S_{N H}(z, \tau), S_{O}(z, \tau)\right) d z\right] r_{N}(\tau) d \tau=r_{N}(t) \\
-r_{N}(t-D)+\int_{t-D}^{t}\left(b_{A}+\frac{Q_{w}(\tau)}{V}\right) r_{N}(\tau) d \tau
\end{gathered}
$$

Integrating the second equation in model (1) in the same way, one has:

$$
\begin{aligned}
\int_{t-D}^{t} v\left(S_{N H}(z, \tau), S_{O}(z, \tau)\right) r_{N}(\tau) d \tau= & S_{N N}(z, t-D)-S_{N N}(z, t) \\
& -\frac{1}{A} \int_{t-D}^{t} Q(\tau) \frac{\partial S_{N N}(z, \tau)}{\partial z} d \tau \\
& -\int_{t-D}^{t} r_{X B}(z, \tau) d \tau
\end{aligned}
$$

Introducing equation (7) into (6), one obtains:

$$
\begin{gathered}
\mu_{A, \max }(t)= \\
\frac{r_{N}(t)-r_{N}(t-D)+\int_{t-D}^{t}\left(b_{A}+\frac{Q_{w}(\tau)}{V}\right) r_{N}(\tau) d \tau}{\frac{1}{l} \int_{0}^{l}\left(S_{N N}(z, t-D)-S_{N N}(z, t)\right) d z+\frac{1}{V} \int_{t-D}^{t} Q(\tau)\left(S_{N N}(0, \tau)\right.} \\
-S_{N N}(l, \tau) d \tau-\frac{1}{l} \int_{0}^{l} \int_{t-D}^{t} r_{X B}(z, \tau) d \tau d z
\end{gathered}
$$

In equation (8):

- $r_{N}(t)$, the nitrification activity of the sludge, can, for instance, be measured by a titrimetric sensor that is able to measure the ammonia concentration as well as the nitrification activity of the sludge (Gernaey et al., 1997; Massone et al., 1998);

- $S_{N N}(0, t)$, the nitrifiable nitrogen concentration at the beginning of the reactor, can be measured by a newly developed titrimetric respirometer (patent pending);

- $S_{N N}(l, t)$, the nitrifiable nitrogen concentration at the end of the reactor, can be measured by any ammonia sensor (virtually all the nitrifiable nitrogen has been converted into ammonia at the end of the reactor);

- The first term in the denominator is small compared with the result of the second and third ones, and can thus be dropped, or be estimated by interpolating $S_{N N}(0, t)$ and $S_{N N}(l, t)$, without introducing significant errors. This is due to the fact that $D$ is usually chosen in an order of days, which will be discussed later;

- The last term in the denominator can be estimated with:

$$
\begin{aligned}
\frac{1}{l} \int_{0}^{l} \int_{t-D}^{t} r_{X B}(z, \tau) d \tau d z= & \frac{1}{l} \int_{0}^{l} \int_{t-D}^{t}\left(i_{X B} \mu_{H}(z, t) X_{H}(t)-\left(i_{X B}\right.\right. \\
& \left.\left.-f_{P} i_{X P}\right) b_{H} X_{H}(t)\right) d \tau d z \\
\approx & D Y_{H} \frac{i_{X B}+\theta_{X} f_{P} b_{H} i_{X P}}{b_{H} \theta_{X}+1} B_{V}
\end{aligned}
$$

where $B_{V}$ is the average volumetric biodegradable COD loading rate to the plant. The second equality in equation (9) is approximate due to the fact that, in the derivation, the system was assumed to be in its steady state: $\left(\int_{0}^{l} \mu_{H}(z, t) d z\right) / l$ $=b_{H}+1 / \theta_{X} ; X_{H}=Y_{H} B_{V} /\left(b_{H}+1 / \theta_{X}\right)$ by assuming a constant $B_{V}$. However, the impact of the $B_{V}$ variation is partially diminished by the integration. For modern plants, where the daily $B_{V}$ is available, the average $B_{V}$ over $[t-D, t]$ should be used in equation (9).

Obviously, equation (8) constitutes an algorithm for estimating $\mu_{A, \max }$, provided that, in addition to the measurements discussed above, the flow rates $Q(t)$ and $Q_{w}(t)$ are measured and that $b_{A}$ and the parameters involved in equation (9) are known. The sensitivity of the algorithm to these parameters will be analyzed in the next section.

By applying a similar transformation to equation (3) and the third equation in model (1), a variant of the algorithm is obtained: 


$$
\begin{gathered}
\mu_{A, \max }(t)= \\
\frac{r_{N}(t)-r_{N}(t-D)+\int_{t-D}^{t}\left(b_{A}+\frac{Q_{w}(\tau)}{V}\right) r_{N}(\tau) d \tau}{\frac{1}{l} \int_{0}^{l}\left(S_{N O}(z, t)-S_{N O}(z, t-D)\right) d z+\frac{1}{V} \int_{t-D}^{t} Q(\tau)\left(S_{N O}(l, \tau)\right.} \\
-S_{N O}(0, \tau) d \tau+\frac{1}{l} \int_{0}^{l} \int_{t-D}^{t} r_{D}(z, \tau) d \tau d z
\end{gathered}
$$

Different from the previous one, nitrate nitrogen, instead of the nitrifiable nitrogen, should be measured at the two ends of the reactor (the two ends of the aerobic part if the reactor has an anoxic zone), in addition to measuring the nitrification activity of the sludge.

One difficulty with this algorithm is to determine the last term in the denominator, i.e., the amount of nitrate that is removed via the denitrification under presumed aerobic conditions during $[t-D, t]$. It depends on many factors, such as the oxygen concentration, the denitrifying heterotrophic biomass concentration, and so on. An experiment which involves monitoring for a few days the nitrifiable nitrogen and COD load to the plant, in addition to the measurement of the nitrate nitrogen concentrations, can be used to calibrate this term. Mass balancing then allows calculating the amount of nitrate removed in the period. The sensitivity of the algorithm to this term will be analyzed in the next section.

\section{DISCUSSION}

\section{Sensitivity of the Algorithm to Model Parameter Variations}

Kinetic parameter $b_{A}$ is involved in both estimator (8) and estimator (10). The sensitivity of the algorithm to this parameter is revealed by the following sensitivity function, obtained based on assuming a steady state of the process:

$$
\frac{\partial \mu_{A, \max , e s t i}}{\partial b_{A}}=\frac{\mu_{A, \max }}{b_{A}+1 / \theta_{X}}
$$

The above sensitivity function is obtained by calculating the partial derivative of the right hand side of equation (8) (or equation (10)) w.r.t. $b_{A}$ and making use of the fact that, in steady state, $r_{N}(t)=r_{N}(t-D)$ and $V / Q_{w}(t)=\theta_{X} \cdot \mu_{A \text {, max,esti }}$ is used here to denote the outcome of equation (8) or (10) to differentiate itself from the actual value of $\mu_{A, \max }$. As a nitrifying plant is such that $\mu_{A \text {, max }}>b_{A}+1 / \theta_{X}$, the error associated with $b_{A}$ will be amplified in the estimation. This is generally true for any $\mu_{A \text {, max }}$ estimation algorithms based on the currently available measurements. Therefore, $b_{A}$ should be carefully calibrated for each individual plant under study. Nowak et al. (1994) presented an approach to calibrating $b_{A}$.

Equation (8) involves five additional stoichiometric and kinetic parameters: $i_{X B}, i_{X P}, Y_{H}, f_{P}$, and $b_{H}$, required for the determination of the amount of nitrogen that is assimilated into biomass cells (the last term of the denominator). The sensitivity of the algorithm to these parameters is revealed by the following sensitivity function (assuming the process runs at steady state):

$$
\frac{\partial \mu_{A, \max , \text { esti }}}{\partial \beta}=\frac{\mu_{A, \max }}{1-\beta}
$$

where $\beta$ denotes the fraction of the assimilated nitrogen (the last term in the denominator of equation (8)) in the totally removed (nitrified and assimilated) nitrifiable nitrogen. As $\beta$ is typically between 0.1 to 0.2 , sensitivity function (12) indicates that an error with $\beta$ caused by inaccurate parameter values will not result in a significant estimation error. For example, assuming $\beta=0.15$ while its real value is 0.1 , which is a significant error $(50 \%)$, will result in an estimation error of $1-6 \%$ when $\mu_{A \text {, max }}$ has a value within 0.3 to 1 . The sensitivity function suggests that the default values recommended in literature (e.g. Henze et al., 1987) for the parameters involved in the assimilation term could be used in the estimation in case their exact values are not known.

To evaluate the sensitivity of estimator (10) to the possible errors associated with the last term of its denominator, a similar analysis is made. Denoting this term as a fraction, say $\gamma$, of all nitrified nitrogen, sensitivity analysis under steady state gives:

$$
\frac{\partial \mu_{A, \max , \text { esti }}}{\partial \gamma}=\frac{\mu_{A, \max }}{1-\gamma}
$$

Obviously, the estimate of $\mu_{A \text {, max }}$ is rather robust to the errors and/or variations associated with $\gamma$ as long as $\gamma$ is small, which is the case for ordinary nitrogen removal plant. Difficulties could be expected when applying estimator (10) to a plant that does simultaneous nitrification and denitrification plant (Münch et al., 1996).

\section{Impact of Measurement Noise and Choice of D}

Assuming that the measurement noise associated with $r_{N}$, $S_{N N}$ and $S_{N O}$ is white Gausian. As $S_{N N}$ and $S_{N O}$ only appear in integration terms in estimators (8) and (10), the random noise associated with these variables has marginal impact on the estimation. The noise associated with $r_{N}$ has the largest impact.

For simplicity, assume that the system runs in steady state. Further assume that $r_{N}$ is measured with a sampling period of $T_{s}$ (about 0.5 to $1 \mathrm{~h}$ when the titrimetric sensor is used (Gernaey et al., 1997; Massone et al., 1998)) and that the measured $r_{N}$, denoted as $r_{N \text {,meas }}$, has the following normal distribution:

$$
r_{N, \text { meas }} \propto N\left(m_{r N}, \sigma_{r N}^{2}\right)
$$

The following distributions can be obtained: 


$$
\begin{aligned}
& r_{N, \text { meas }}(t)-r_{N, \text { meas }}(t-D)+\int_{t-D}^{t}\left(b_{A}+\frac{V}{Q_{w}}\right) r_{N, \text { meas }}(\tau) d \tau \\
& \propto N\left(\left(b_{A}+\frac{1}{\theta_{X}}\right) D m_{r N},\left(2+\frac{b_{A}+1 / \theta_{X}}{D / T_{s}^{2}}\right) \sigma_{r N}^{2}\right)
\end{aligned}
$$

The impact of $D$ on the estimation accuracy is twofold:

- A larger $D$ tends to reduce the covariance of the above distribution. However, this impact appears to be minor as the covariance approaches $2 \sigma_{r N}^{2}$, its minimum value, when $D>>T_{s}$, which will be case. The minimum covariance can be reduced if the average of a few data points around $t$ and $t-D$ are used to replace a single measured value of $r_{N}(t)$ and $r_{N}(t-D)$ in estimators (8) and (10). When the average of $M$ data points are used, the minimum covariance is reduced to $2 \sigma_{r N}^{2} / M$.

- The mean value of the distribution in (15) is proportional to $D$, which means that a larger $D$ reduces the relative error caused by the measurement error. Note that the covariance does not increase with $D$.

On the other hand, $D$ should be designed as small as possible in order to track the variations of $\mu_{A, \max }$. Given the measurement accuracy of the existing sensors, $D$ should be in the range of one to a few days. As such, the developed estimation algorithm is not suitable for detecting abrupt changes of $\mu_{A, \max }$. This is, however, not a serious problem because an abrupt change of $\mu_{A, \max }$ can readily be detected by investigating the $r_{N}$ measurement itself (see equation (5)). Under the reasonable assumption of $Y_{A}$ and $X_{A}$ being constant over a short period, $r_{N}$ has been suggested as an indication of an abrupt change in $\mu_{A, \max }$ (Vanrolleghem et al., 1994, 1996).

\section{Compensation of the Temperature Effects}

The assumption that $\mu_{A, \max }$ remains constant in $[t-D, t]$ may not be true when the temperature of the mixed liquor in the plant is subject to significant variations due to, for instance, air temperature variations, rainfall, or snow melting events, as this results in significant changes of $\mu_{A \text {, max }}$. Temperature compensation is needed in such circumstances.

The following relationship has been well established to characterize how temperature influences the maximum specific growth rate of nitrifiers (see, e.g., EPA, 1993):

$$
\mu_{A, \max , T}=\mu_{A, \max , T_{0}} e^{\alpha\left(T-T_{0}\right)}
$$

where $\mu_{A, \max , T}$ denotes the maximum specific growth rate of nitrifiers at temperature $T . T_{0}$ is a reference temperature, which is typically, but not necessarily, chosen as $15^{\circ} \mathrm{C} . \alpha$ is the unique coefficient involved in the function, which can be calibrated by experiments (a procedure for calibrating $\alpha$ is explained below). EPA (1993) recommends a value of 0.098 for this parameter. Our experiments (data not shown) suggested a similar value.

By multiplying $X_{A} / Y_{A}$ on both sides of equation (16) the following relationship is obtained:

$$
r_{N, T}=r_{N, T_{0}} e^{\alpha\left(T-T_{0}\right)}
$$

where $r_{N, T}$ denotes the nitrification activity of a sludge at temperature $T$.

In analogy to the procedure used in deriving equation (8), the following equation can be obtained:

$$
\begin{array}{r}
\mu_{A, \max , T_{0}}= \\
\frac{r_{N, T_{0}}(t)-r_{N, T_{0}}(t-D)+\int_{t-D}^{t}\left(b_{A}+\frac{Q_{w}(\tau)}{V}\right) r_{N, T_{0}}(\tau) d \tau}{\frac{1}{l} \int_{0}^{l}\left(S_{N N}(z, t-D)-S_{N N}(z, t)\right) d z+\frac{1}{V} \int_{t-D}^{t} Q(\tau)\left(S_{N N}(0, \tau)\right.} \\
\quad-S_{N N}(l, \tau) d \tau-\frac{1}{l} \int_{0}^{l} \int_{t-D}^{t} r_{X B}(z, \tau) d \tau d z
\end{array}
$$

where $r_{N, T 0}(\tau), \tau \in[t-D, t]$ can be calculated from $r_{N, T}(\tau)$ by reversing equation (17). Obviously, the implicit assumption behind equation (18) is that the maximum specific growth rate of nitrifiers at the reference temperature $T_{0}$ remains a constant during time interval $[t-D, t]$, which is more justified. Equation (18) gives the maximum specific growth rate of nitrifiers at temperature $T_{0}$. This rate at time $t$, with a temperature $T(t)$, can be obtained using equation (17).

The calibration of parameter $\alpha$ can be done using equation (17). Plotting the activities of the same sludge sample measured at different temperatures versus the temperatures will yield the parameter value.

It is well known that $b_{A}$ also depends on temperature (Nowak, 1996). A similar correction for $b_{A}$ can also be included in (18).

\section{Elimination of the Impact of the Settler Dynamics}

A point settler model was assumed in deriving the algorithm. In practice, however, this is often invalid since there is always some sludge stored in the settler with an amount determined by the hydraulic loading to the plant, the sludge recycling rate, and the sludge settleability. During wet weather periods, up to $30-50 \%$ of the total sludge may reside in the settler. Due to the fact that the sludge is unevenly distributed below the blanket in a settler, a spatial sampling in the settler is usually needed to quantify the stored amount of nitrifiers, which is expensive to realize. With the algorithm developed above, this problem can be circumvented, as shown below.

When the settler dynamics is taken into consideration, the nominator of equations (8) and (10) becomes:

$$
\begin{aligned}
& r_{N}(t)-r_{N}(t-D)+\int_{t-D}^{t}\left(b_{A}+\frac{Q_{w}(\tau)}{V}\right) r_{N}(\tau) d \tau \\
& +\left(\int_{t-D}^{t} \frac{\left(Q_{\mathrm{inf}}(\tau)+Q_{r}(\tau)\right)}{V} r_{N}(\tau) d \tau-\int_{t-D}^{t} \frac{Q_{r}(\tau)}{V} r_{N, r}(\tau) d \tau\right)
\end{aligned}
$$

where $r_{N, r}$ is the nitrification activity of the recycled sludge. The last term in the above equation is the increment of the total nitrification capacity stored in the settler during $[t-D, t]$. 
As is known, the amount of sludge residing in the settler is mainly determined by the hydraulic flows to and from the settler when a short period is considered. In other words, the amount of sludge in the settler would be approximately the same at time $t-D$ and $t$ if the settler experiences similar flows around the two moments. This allows the elimination of the last term by on-line adjusting $D$ such that both the influent flow and the recycling flow are approximately the same around moments $t-D$ and $t$. An example for the adjustment of $D$ will be given in the simulation study (below).

\section{MATERIALS AND METHODS}

The experimental study was conducted on a pilot plant, shown schematically in Figure 2. It is a predenitrification plant with four cascaded completely mixed tanks. The first is anoxic and the rest are aerobic. The dimensions of the plant are also shown in the figure. The plant was fed with synthetic influent (milk powder + ammonia chloride). Constant influent, sludge recycling, nitrate recirculation, and surplus sludge waste flow rates were applied throughout the experiment, which were $11 \mathrm{~L} / \mathrm{h}, 8 \mathrm{~L} / \mathrm{h}, 36 \mathrm{~L} / \mathrm{h}$, and $1.25 \mathrm{~L} / \mathrm{h}$, respectively. A constant influent COD concentration of about $675 \mathrm{mgCOD} / \mathrm{L}$ was applied throughout the experiment, while the influent TKN concentration was raised from $57 \mathrm{mgN} / \mathrm{L}$ to $118 \mathrm{mgN} / \mathrm{L}$ during the last 3 days of the experiment (see Fig. 3).

A titrimetric sensor (Massone et al., 1998) was used to measure the nitrification activity of the sludge. As the sludge samples were taken manually from the plant, $r_{N}$ could only be measured during the daytime. Constant influent was therefore applied to the plant to avoid stimulating fast variations of $r_{N}$. The results are shown in Figure 3. The mixed liquor temperature was also followed during the experiment (Fig. 6).

An on-line ammonia analyzer (Minworth Systems Ltd., Sutton Coldfield, UK) was used to measure the ammonia concentration in the effluent of the pilot plant. The analyzer was operated on the effluent to avoid the need for an expensive ultrafiltration unit which would be necessary for sample pretreatment when operating the analyzer immediately on the mixed liquor. As the settler is rather small (10 L), it does not make much difference measuring the effluent or the last aerobic zone. The data are shown in Figure 3.

Simulation studies were also performed to evaluate the algorithm. Compared with experimental studies, simulation studies allow evaluating the algorithms under much more

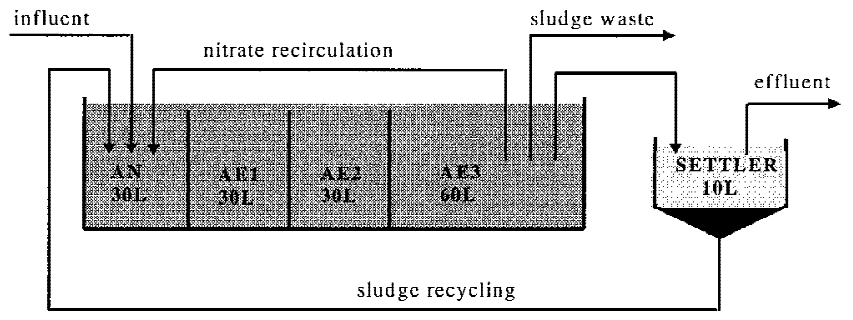

Figure 2. Structure and dimensions of the pilot plant.

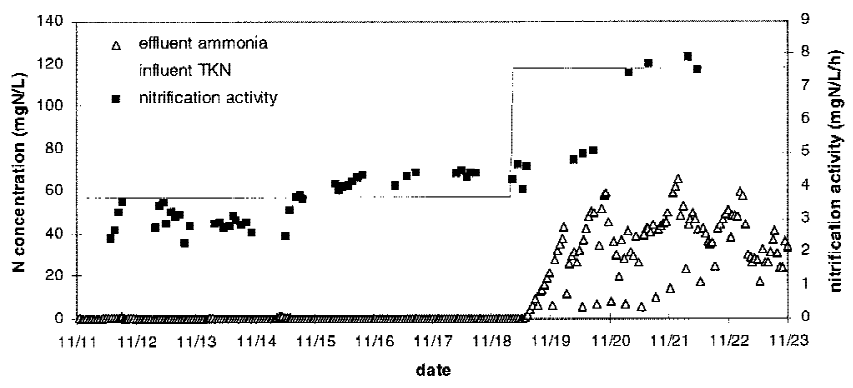

Figure 3. Measured nitrification activity, effluent ammonia concentration, and known influent TKN concentration.

complicated circumstances. Also, they allow verifying the results since the "real" parameter values are known.

The pilot plant shown in Figure 2 was used in the simulation study. Each tank was modeled with the IAWQ model no. 1 (Henze et al., 1987). The real values of $\mu_{A \text {, max }}$ applied in the simulation is shown in Figure 8. Other parameters take values as recommended in Henze et al. (1987). The settler was modeled with a 10 layer Takács model (Takács et al., 1991). The settler was assumed to be $50 \mathrm{~L}$, as a $10 \mathrm{~L}$ settler is too small to cope with the high hydraulic load applied in the simulation. The influent data (the flow rate, the nitrogen, and the bCOD concentrations) were derived by down-scaling the real influent data of a full-scale wastewater treatment plant in Flanders, Belgium. The influent flow rate is shown in Figure 4. The flow rate varied from 100 $\mathrm{L} \cdot \mathrm{d}^{-1}$ to as high as about $600 \mathrm{~L} \cdot \mathrm{d}^{-1}$, with an average of approximately $200 \mathrm{~L} \cdot \mathrm{d}^{-1}$. Similar variations were also observed in the nitrogen and bCOD loading rates to the plant (Fig. 5). Constant nitrate recirculation and surplus sludge waste flow rates were used $\left(Q_{i n t}=800 \mathrm{~L} \cdot \mathrm{d}^{-1}, Q_{w}=15\right.$ $\left.\mathrm{L} \cdot \mathrm{d}^{-1}\right)$. The sludge recycling flow rate was controlled to be proportional to the influent flow rate (ratio $=1$ ) with a maximum rate being $250 \mathrm{~L} \cdot \mathrm{d}^{-1}$.

In the simulation study, the following measurement noise was introduced: $e\left(r_{N}\right) \propto \mathrm{N}\left(0,7^{2}\right), \quad e\left(S_{N N}\right) \propto \mathrm{N}\left(0,0.25^{2}\right)$, $e\left(S_{N O}\right) \propto \mathrm{N}\left(0,0.25^{2}\right)$.

\section{RESULTS AND DISCUSSION}

\section{Experimental Study}

Given the fact that the effluent ammonia concentration is measured and that the influent TKN is known, since syn-

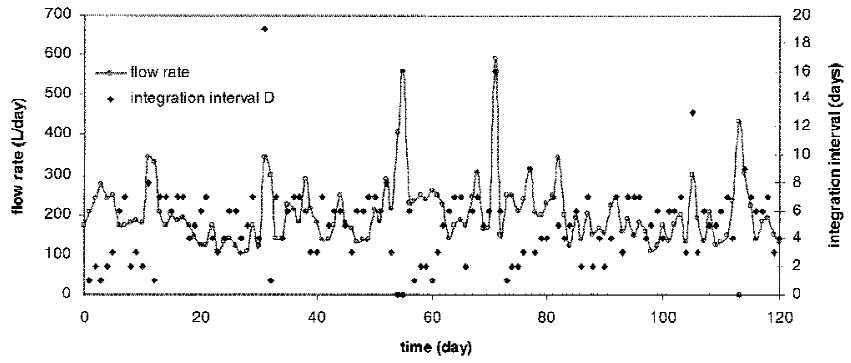

Figure 4. Influent flow rate and the integration intervals $(D)$. 


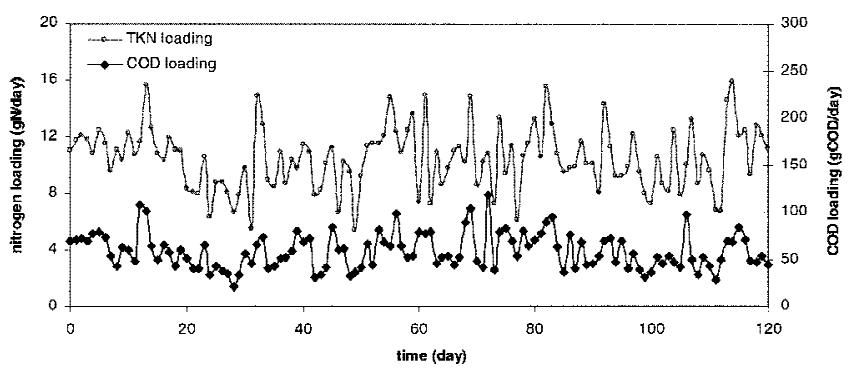

Figure 5. The influent bCOD and TKN loading.

thetic influent is used, equation (8) could be transformed to (it is assumed that all the Kjeldahl nitrogen contained in the synthetic influent is nitrifiable):

$$
\begin{array}{r}
\mu_{A, \max , T_{0}}= \\
\frac{r_{N, T_{0}}(t)-r_{N, T_{0}}(t-D)+\int_{t-D}^{t}\left(b_{A}+Q_{w}(\tau) / V\right) r_{N, T_{0}}(\tau) d \tau}{\left(I(t-D)-I(t)+\int_{t-D}^{t} \frac{Q_{\text {inf }}(\tau)}{V}\left(S_{K N, \text { inf }}(\tau)-S_{N H, \text { eff }}(\tau)\right) d \tau\right.} \\
\left.-D Y_{H} \frac{i_{X B}+\theta_{X} f_{P} b_{H} i_{X P}}{b_{H} \theta_{X}+1} B_{V}\right)
\end{array}
$$

making use of the fact that term $Q(\tau)\left(S_{N N}(0, \tau)-S_{N N}(1, \tau)\right)$ in equation (8) is equal to $Q_{\text {inf }}(\tau)\left(S_{K N \text {,inf }}(\tau)-S_{N H, \text { eff }}(\tau)\right) . V=$ $150 \mathrm{~L}$ is the total volume of the reactor; $T_{0}=15^{\circ} \mathrm{C} ; b_{A}, b_{H}$, $i_{X B}, i_{X P}, Y_{H}$, and $f_{P}$ take their default values as recommended in Henze et al. (1987), and:

$$
\begin{aligned}
I(t) & =\frac{1}{l} \int_{0}^{l} S_{N N}(z, t) d z \\
& \approx \frac{Q_{\mathrm{inf}}(t)}{Q(t)} S_{K N, \mathrm{inf}}(t)+\left(\frac{Q_{\mathrm{int}}(t)+Q_{r}(t)}{Q(t)}+1\right) S_{N H, \text { eff }}(t)
\end{aligned}
$$

where the second approximate equality is obtained by linearly interpolating $S_{N N}(0, t)$ and $S_{N N}(l, t)$ along the length of the reactor. Using equation (20) and the temperature compensation equation (16), $\mu_{A, \max }$ is estimated each day. The results are shown in Figure 6.

To validate these results, $\mu_{A \text {,max }}$ values were calculated with another method as well. Since the pilot plant had been running at steady state for a couple of months before this experiment started, this allowed us to "reliably" estimate the nitrifier concentration based on the nitrogen load to the

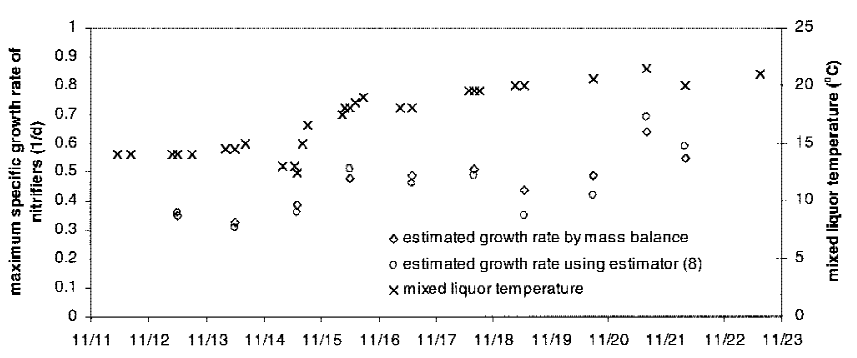

Figure 6. Estimated $\mu_{A, \max }$ and mixed liquor temperature. plant using the default model parameters as recommended in Henze et al. (1987). A second set of $\mu_{A \text {, max }}$ values was thus obtained based on the measured nitrification activities and the estimated nitrifier concentrations. The results are shown in Figure 6. The two sets of estimates are reasonably close to each other, especially for the first 6 days. The relatively larger errors during the last 4 days are probably due to a too small number of activity measurements during these days. Note that the nitrifiers underwent an exponential growth in this period (excess $\mathrm{NH}_{3}$ ).

On November 18 and 19, there was a drop of $\mu_{A, \max }$ despite an increased temperature. This was probably caused by substrate inhibition due to a high ammonia concentration in the reactor. Indeed, substrate inhibition was also observed by independent batch experiments with sludge samples taken from the plant: the nitrification rate significantly increased when the ammonia concentration dropped to a few milligrams per liter from a higher level. Interestingly, substrate inhibition no longer existed on November 20 and 21, although the ammonia concentration remained at a high level, pointing to adaptation of the sludge to the new conditions.

\section{Simulation Study}

Both estimators were used to estimate $\mu_{A \text {,max }}$. All the model parameters involved were assumed known. For estimator (8), the daily average $B_{V}$ load is assumed known. For estimator (10), it was assumed that $20 \%$ of the nitrate was removed in aerobic reactors $(\gamma=0.2)$.

The integration interval $D$ at time $t$ was determined by minimizing the following cost function with the constraint $D \in[1,7]:$

$$
\begin{aligned}
\operatorname{cost}(t, D) & =\left|\left(Q_{\mathrm{inf}, 3 h}(t)-Q_{\mathrm{inf}, 3 h}(t-D)\right)\right| / D \text { if }\left(Q_{\text {inf }, 3 h}(t)\right. \\
& \left.\leq Q_{r, \max } \& Q_{\mathrm{inf}, 3 h}(t-D) \leq Q_{r, \max }\right) \text { or } \\
& \left|Q_{\text {inf }, 3 h}(t)-Q_{\mathrm{inf}, 3 h}(t-D)\right| \leq 0.2 \times Q_{\mathrm{inf}, 3 h}(t) \\
& =\infty \text { otherwise }
\end{aligned}
$$

where $Q_{\text {inf,3h }}(t)$ denotes the 3-h average influent flow rate at time $t ; Q_{r, \text { max }}=250 \mathrm{~L} \cdot \mathrm{d}^{-1}$ is the maximum RAS flow rate. The definition of the cost function is graphically depicted in Figure 7. As has been stated in a previous section, the idea is to choose $D$ such that the settler contains a similar amount

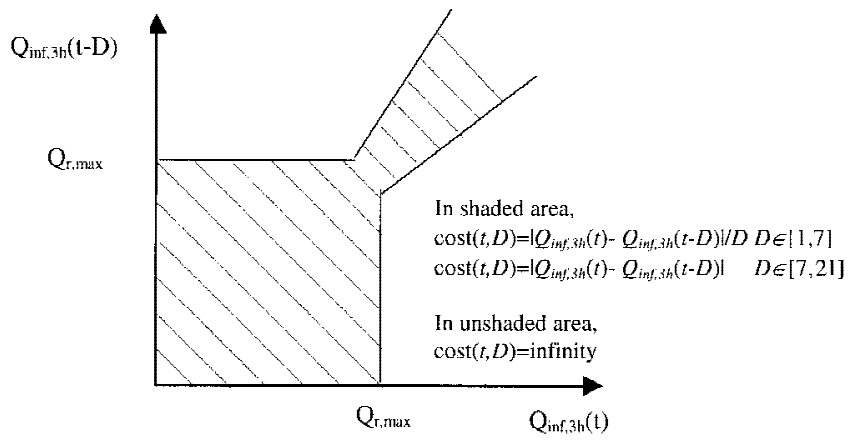

Figure 7. Definition of the cost function for the determination of the integration interval in the simulation study. 
of sludge at $t$ and $t-D$. When $\left(Q_{\mathrm{inf}, 3 h}(t), Q_{\mathrm{inf}, 3 h}(t-D)\right)$ is located in the shaded area, the RAS concentration at $t$ is believed to be approximately the same as that at $t-D$, and the settler likely contains a similar amount of sludge at the two moments. The cost is defined to be proportional to the difference of the 3-h average flow rates at the two moments. A small $D$ is penalized in the cost function in order to improve the estimation accuracy. On the contrary, the settler likely contains significantly different amounts of sludge at $t$ and $t$ - $D$ when $\left(Q_{\text {inf }, 3 h}(t), Q_{\text {inf }, 3 h}(t-D)\right)$ is located in the unshaded area. An infinite cost is thus defined for such circumstances.

If no $D \in[1,7]$ has a finite cost, the optimization is extended to $D \in[7,21]$, with the cost function defined similar to that shown in (22) but without penalizing smaller $D$ (see Fig. 7). $D=0$ is returned if a finite cost is still not obtained. In this case, the estimate of $\mu_{A \text {, max }}$ is not updated.

The estimated $\mu_{A, \max }$ by both estimators are shown in Figure 8. For comparison, the estimated $\mu_{A \text {, max }}$ based on predicting the nitrifier concentration (Nowak et al., 1994) is also shown.

It is found that both estimator (8) and estimator (10) gave reasonably good estimates despite the large variations of the influent flow rate and the substrate loads. It is clear that the estimators are robust to these variations. The proposed algorithm obviously outperformed the nitrifier concentration prediction approach during the periods following accidental sludge losses. On Days 55 and 71, significant amounts of sludge were washed out from the settler due to too-high hydraulic flows to the settler, causing incorrectly predicted nitrifier concentrations. Each time it took more than 2 weeks for the predictor to converge. In contrast, the presented algorithm converged in just 1 day. The same happened at the beginning of the estimation, where the presented algorithms started providing the "right" estimates after 1 day, while it took more than 1 week for the nitrifier prediction approach to start up due to an inaccurate initial condition for the predictor.

Simulation showed that the real fraction of the nitrate that was denitrified in the aerobic zone $(\gamma)$ varied between $5 \%$ and $30 \%$, with an average of $16 \%$. Assuming this fraction as $20 \%$ did not introduce significant errors into estimator (10). The error caused by the short-term variations of $\gamma$ was compensated by the integration, while the error caused by the biased assumption of $\gamma$ ( $\gamma$ assumed to be $20 \%$ instead of its

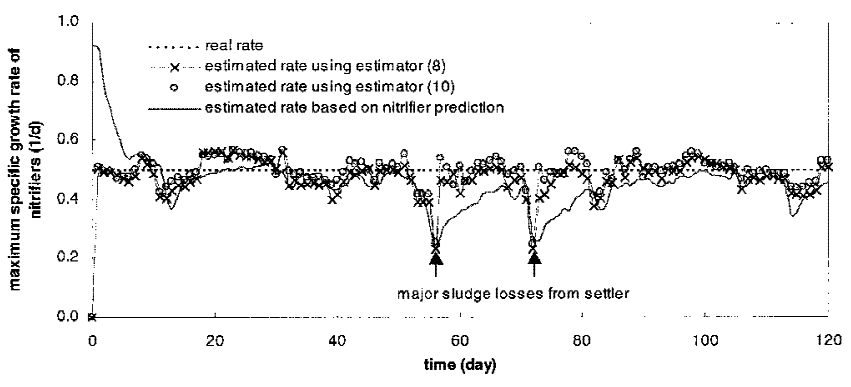

Figure 8. The "real" and estimated $\mu_{A, \max }$ in the simulation study. average value $16 \%$ ) is not clearly visible either. According to sensitivity function (13), the error caused by the biased assumption could only cause an estimation error of about $2 \%$.

The integration intervals used in the estimations are shown in Figure 4. On three occasions, $D$ has a value of 0 , indicating a failure of finding an appropriate $D$. During these 3 days the influent flow rate was extremely high, so that a matching flow could not be found within 3 weeks.

An inherent disadvantage of the developed algorithm is that it is only capable of estimating the average $\mu_{A \text {, max }}$ over a time interval $([t-D, t])$. As such, the estimates do not reflect the instant change of $\mu_{A \text {, max }}$, as does the nitrifier concentration prediction approach. Note that since the nitrifier prediction approach requires exactly the same information as the developed approach in this study, it is advisable to use the two approaches in parallel when the instant change of $\mu_{A \text {, max }}$ is of interest. A large deviation between the two estimates will reinitiate the nitrifier concentration predictor, with the initial concentration being determined based on the measured nitrification activity, the estimated $\mu_{A, \max }$ by the presented algorithm and an assumed $Y_{A}$.

\section{CONCLUSIONS}

The on-line estimation of the maximum specific growth rate of autotrophic biomass has been studied. An algorithm was developed, which requires measuring the nitrification activity of the sludge, and the nitrifiable nitrogen or nitrate nitrogen concentrations at the two ends of the bioreactor.

The following conclusions are drawn.

- Unlike most of the existing on-line algorithms for $\mu_{A \text {, max }}$ estimation, which rely on the assumption that the plant has ideal flow patterns (completely mixed bioreactors and point settlers), the developed algorithm is applicable to plants with any realistic flow patterns.

- The algorithm has a short start-up period and recovers soon from accidental sludge losses. This is attributed to the fact that it does not require estimating the nitrifier concentration, which converges slowly.

- The developed algorithm, like any other $\mu_{A, \max }$ estimation algorithm, is sensitive to the autotrophic decay rate. An effort should be made to calibrate the autotrophic decay rate accurately. Other parameters involved in the estimation equations have limited influence on the estimation results, and therefore could take their default values as recommended in the literature.

\section{NOMENCLATURE}

$A$
$b_{A}$
$b_{H}$
$B_{V}$

$e\left(S_{N N}\right)$
$e\left(S_{N O}\right)$
$e\left(r_{N}\right)$

sectional area of a plug flow reactor $\left(\mathrm{m}^{2}\right)$

decay coefficient of autotrophic biomass $\left(\mathrm{d}^{-1}\right)$

decay coefficient of heterotrophic biomass $\left(\mathrm{d}^{-1}\right)$

volumetric biodegradable COD loading rate to the plant $\left(\mathrm{gCOD} \cdot \mathrm{m}^{-3} \cdot \mathrm{d}^{-1}\right)$

noise associated with the measurement of $S_{N N}\left(\mathrm{gN} \cdot \mathrm{m}^{-3}\right)$ noise associated with the measurement of $S_{N O}\left(\mathrm{gN} \cdot \mathrm{m}^{-3}\right)$ noise associated with the measurement of $r_{N}\left(\mathrm{gN} \cdot \mathrm{m}^{-3}\right.$. 


\begin{tabular}{|c|c|}
\hline & $\left.d^{-1}\right)$ \\
\hline$D$ & integration interval (d) \\
\hline$p$ & fraction of biomass leading to inert products $(-)$ \\
\hline$X B$ & $\begin{array}{l}\text { fraction of ammonia nitrogen in biomass }(\mathrm{gN} \cdot(\mathrm{g} \text { cell } \\
\left.\left.\mathrm{COD})^{-1}\right)\right)\end{array}$ \\
\hline$i_{X P}$ & $\begin{array}{l}\text { fraction of ammonia nitrogen in endogenous mass }(\mathrm{gN} \cdot \\
\left.(\mathrm{gCOD} \text { endogenous mass })^{-1}\right)\end{array}$ \\
\hline & length of a plug flow reactor $(\mathrm{m})$ \\
\hline$Q(t)$ & $\begin{array}{l}\text { flow rate flared to the reactor }\left(\mathrm{m}^{3} \cdot \mathrm{d}^{-1}\right) \cdot Q(t)=Q_{\mathrm{inf}}(t)+ \\
Q_{r}(t) \text {, or for predentrification system, } Q(t)=Q_{\mathrm{inf}}(t)+Q_{r}(t) \\
+Q_{\mathrm{int}}(t) \text {. }\end{array}$ \\
\hline$Q_{\text {inf }}(t)$ & influent flow rate $\left(\mathrm{m}^{3} \cdot \mathrm{d}^{-1}\right)$ \\
\hline$Q_{\mathrm{inf}, 3 \mathrm{~h}}(t)$ & influent flow rate averaged over $3 \mathrm{~h}\left(\mathrm{~m}^{3} \cdot \mathrm{d}^{-1}\right)$ \\
\hline$Q_{\text {int }}(t)$ & nitrate recirculation flow rate $\left(\mathrm{m}^{3} \cdot \mathrm{d}^{-}\right.$ \\
\hline$Q_{r}(t)$ & sludge recycling flow rate $\left(\mathrm{m}^{3} \cdot \mathrm{d}^{-1}\right)$ \\
\hline$Q_{r, \text { max }}(t)$ & maximum capacity of the sludge recycling pump $\left(\mathrm{m}^{3} \cdot \mathrm{d}^{-1}\right)$ \\
\hline$Q_{w}(t)$ & surplus sludge waste flow rate $\left(\mathrm{m}^{3} \cdot \mathrm{d}^{-1}\right)$ \\
\hline$r_{D}(z, t)$ & $\begin{array}{l}\text { volumetric nitrate reduction rate due to denitrification under } \\
\text { aerobic conditions }\left(\mathrm{gN} \cdot \mathrm{m}^{-3} \cdot \mathrm{d}^{-1}\right)\end{array}$ \\
\hline$r_{N}(t)$ & nitrification activity of the sludge $\left(\mathrm{gN} \cdot \mathrm{m}^{-3} \cdot \mathrm{d}^{-1}\right)$ \\
\hline$r_{N, \text { meas }}(t)$ & measured nitrification activity of the sludg \\
\hline$r_{N, r}(t)$ & nitrification activity of the rec \\
\hline$r_{X B}(z, t)$ & $\begin{array}{l}\text { volumetric ammonia consumption rate due to the growth of } \\
\text { biomass }\left(\mathrm{gN} \cdot \mathrm{m}^{-3} \cdot \mathrm{d}^{-1}\right)\end{array}$ \\
\hline$S_{K N, \text { inf }}(z, t)$ & influent Kjeldahl nitrogen concentration $\left(\mathrm{gN} \cdot \mathrm{m}^{-3}\right)$ \\
\hline$S_{N H}(z, t)$ & $\begin{array}{l}\text { ammonia nitrogen concentration at position } z \text { and time } t \\
\left(\mathrm{gN} \cdot \mathrm{m}^{-3}\right)\end{array}$ \\
\hline$S_{N H, e f f}(t)$ & effluent ammonia nitrogen concentration $\left(\mathrm{gN} \cdot \mathrm{m}^{-3}\right)$ \\
\hline$S_{N O}(z, t)$ & $\begin{array}{l}\text { nitrate nitrogen concentration at position } z \text { and time } t \\
\left(\mathrm{gN} \cdot \mathrm{m}^{-3}\right)\end{array}$ \\
\hline$S_{N O, \text { inf }}(z, t)$ & influent nitrate nitrogen concentration $\left(\mathrm{gN} \cdot \mathrm{m}^{-3}\right)$ \\
\hline$S_{N N \text {,inf }}(t)$ & entration $\left(\mathrm{gN} \cdot \mathrm{m}^{-3}\right)$ \\
\hline$S_{N N}(z, t)$ & $\begin{array}{l}\text { nitrifiable nitrogen concentration at position } z \text { and time } t \\
\left(\mathrm{gN} \cdot \mathrm{m}^{-3}\right)\end{array}$ \\
\hline$S_{O}(z, t)$ & oxygen concentration at position $z$ and time $t\left(\mathrm{gN} \cdot \mathrm{m}^{-3}\right)$ \\
\hline$T$ & \\
\hline$T_{0}$ & reference temperature $\left({ }^{\circ} \mathrm{C}\right)$ \\
\hline$T_{s}$ & sampling period (d) \\
\hline$X_{A}(z, t)$ & $\begin{array}{l}\text { autotrophic biomass concentration at position } z \text { and time } t \\
\left((\mathrm{~g} \text { cell } \mathrm{COD}) \cdot \mathrm{m}^{-3}\right)\end{array}$ \\
\hline$X_{A}(t)$ & $\begin{array}{l}\text { average concentration of autotrophic biomass in the reactor } \\
\text { at time } t\left((\mathrm{~g} \text { cell } \mathrm{COD}) \cdot \mathrm{m}^{-3}\right)\end{array}$ \\
\hline$X_{H}(t)$ & $\begin{array}{l}\text { average concentration of heterotrophic biomass in the reac- } \\
\text { tor at time } t\left((\mathrm{~g} \text { cell COD }) \cdot \mathrm{m}^{-3}\right)\end{array}$ \\
\hline$Y_{A}$ & $\begin{array}{l}\text { yield coefficient of autotrophic biomass ( }(\mathrm{g} \text { cell COD } \\
\left.\text { formed }) \cdot(\mathrm{gN} \text { oxidized })^{-1}\right)\end{array}$ \\
\hline$Y_{H}$ & $\begin{array}{l}\text { yield coefficient of heterotrophic biomass ( }(\mathrm{g} \text { cell COD } \\
\left.\text { formed }) \cdot(\mathrm{g} \text { COD oxidized })^{-1}\right)\end{array}$ \\
\hline & th of a plugflow reactor $(\mathrm{m})$ \\
\hline$\beta$ & $\begin{array}{l}\text { fraction of the assimilated nitrogen in the totally removed } \\
\text { nitrifiable nitrogen }(-)\end{array}$ \\
\hline$y$ & $\begin{array}{l}\text { fraction of the nitrate nitrogen denitrified 'aerobically' in } \\
\text { the total nitrified nitrogen }(-)\end{array}$ \\
\hline$\Theta_{X}$ & sludge age (d) \\
\hline$\mu_{A, \mathrm{~m}}$ & maximum specific growth rate of autotrophic biomass $\left(\mathrm{d}^{-1}\right)$ \\
\hline$\mu_{A, \text { max,est }}$ & $\begin{array}{l}\text { estimated maximum specific growth rate of autotrophic bio- } \\
\text { mass }\left(\mathrm{d}^{-1}\right)\end{array}$ \\
\hline$\mu_{A}(z, t)$ & $\begin{array}{l}\text { specific growth rate of autotrophic biomass at position } z \text { and } \\
\text { time } t\left(\mathrm{~d}^{-1}\right)\end{array}$ \\
\hline
\end{tabular}

\section{References}

Antoniou P, Hamilton J, Koopman B, Jain R, Holloway B, Lyberatos G, Svoronos SA. 1990. Effect of temperature and $\mathrm{pH}$ on the effective maximum specific growth rate of nitrifying bacteria. Water Res 24(1): 97-101.

Beccari M, Marani D, Ramadori R. 1979. A critical analysis of nitrification alternatives. Water Res 13(2):185-192.

Beck MB. 1981. Operational estimation and prediction of nitrification dynamics in the activated sludge process. Water Res 15(12): 1313-1330.

EPA. 1993. Manual nitrogen control. US Environmental Protection Agency, EPA/625/R-93/010, Sept. 1993.

Gernaey K, Bogaert H, Massone A, Vanrolleghem P, Verstraete W. 1997. On-line nitrification monitoring in activated sludge with a titrimetric sensor. Environ Sci Technol 31:2350-2355.

Gernaey K, Vanrolleghem P, Verstraete W. 1998. On-line estimation of kinetic parameters of Nitrosomonas kinetic parameters in activated sludge samples using titration in-sensor-experiments. Water Res 32(1): 71-80.

Hall IR. 1974. Some studies on nitrification in the activated sludge process. Water Pollut Control 73:538-546.

Hall ER, Murphy KL. 1980. Estimation of nitrifying biomass and kinetics in wastewater. Water Res 14(4):297-304.

Henze M, Grady CPL Jr, Gujer W, Marais GvR, Matsuo T. 1987. Activated sludge model no. 1, IAWPRC scientific and technical reports no. 1. Bristol, UK: J. W. Arrowsmith Ltd.

Kabouris JC, Georgakakos AP. 1996. Parameter and state estimation of the activated sludge process-II. Applications. Water Res 30(12): $2867-2882$.

Larrea L, Garcia-Heras JL, Ayesa E, Florez F. 1992. Designing experiments to determine the coefficients of activated sludge models by identification algorithms. Water Sci Tech 25(6):149-165.

Marsili-Libelli S, Giovannini F. 1997. On-line estimation of the nitrification process. Water Res 31(1):179-185.

Massone A, Gernaey K, Rozzi A, Verstraete W. 1998. Measurement of ammonium concentration and nitrification rate by a new titrimetric biosensor. Water Environ Res 70(3):343-350.

Münch EV, Lant P, Keller J. 1996. Simultaneous nitrification and denitrification in bench-scale sequencing batch reactors. Water Res 30(2): 277-284.

Nowak O. 1996. Nitrifikation im belebungsverfahren bei maßgebendem industrieabwassereinfluß. Ph.D. thesis. Institut für Wassergüte und Abfallwirtschaft TU-Wien.

Nowak O, Svardal K. 1993. Observations on the kinetics of nitrification under inhibiting conditions caused by industrial water compounds. Water Sci Tech 28(2):115-123.

Nowak O, Schweighofer P, Svardal K. 1994. Nitrification inhibition-a method for the estimation of actual maximum autotrophic growth rates in activated sludge systems. Water Sci Tech 30(6):9-19.

Sözen S, Orhon D, San HA. 1996. A new approach for the evaluation of the maximum specific growth rate in nitrification. Water Res 30(7): 1661-1669.

Spanjers H, Vanrolleghem P. 1995. Respirometry as a tool for rapid characterisation of wastewater and activated sludge. Water Sci Tech 31(2): 104-114.

Srinath E, Prakasam T, Loehr R. 1976. Nitrifying organism concentration and activity. J Environ Engin 102(2):449-463.

Takács I, Patry G, Nolasco D. 1991. A dynamic model of the clarificationthickening process. Water Res 25(10):1263-1271.

Vanrolleghem PA, Kong Z, Rombouts G, Verstraete W. 1994. An on-line respirographic biosensor for the characterization of load and toxicity of wastewaters. J Chem Technol Biotechnol 59:321-333.

Vanrolleghem PA, Kong Z, Coen F. 1996. Full-scale on-line assessment of toxic wastewaters causing change in biodegradation model structure and parameters. Water Sci Tech 33(2):163-175. 